
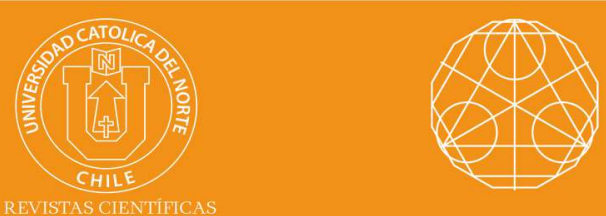

\title{
A certain subclass of uniformly convex functions defined by Bessel functions
}

P. Thirupathi Reddy* iD orcid.org/0000-0003-49095269

B. Venkateswarlu** (iD) orcid.org/0000-0003-3669-350X

*Kakatiya University, Dept. of Mathematics, Warangal, TG, India.

$\checkmark$ reddypt2@gmail.com

${ }^{* *}$ GITAM University, Dept. of Mathematics,GST, Doddaballapur, KA, India.

v bvlmaths@gmail.com

Received: June 2018 | Accepted: October 2019

\section{Abstract:}

The aim of the present paper is to investigate some characterization for generalized Bessel functions of the first kind is to be a subclass of analytic functions. Furthermore, we studied coefficient estimates, radius of starlikeness, convexity, close - to convexity, convex linear combinations for the class $T S(\lambda, \gamma)$. Finally we proved Integral means inequalities for the class.

Keywords: Analytic function; Bessel function; Starlike; Subordination and Hadamard product.

$\operatorname{MSC}(2010): 30 C 45$.

\section{Cite this article as (IEEE citation style)}

R. Dhar, "Some pairwise weakly Fuzzy mappings", Proyecciones (Antofagasta, On line), vol. 38, no. 4, pp. 719-731, Oct. 2019 , doi: 10.22199/issn.0717-6279-2019-04-0046. [Accessed ddmm-yyyy].

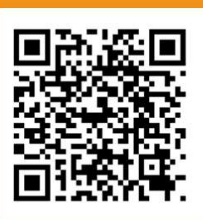

Article copyright: (C) 2019 Pinninti Thirupathi Reddy and Bolineni Venkateswarlu. This is an open access article distributed under the terms of the Creative Commons Licence, which permits unrestricted use and distribution provided the original author and source are credited. 


\section{Introduction}

Let $A$ be the class of functions $f$ normalized by

$$
f(z)=z+\sum_{n=2}^{\infty} a_{n} z^{n}
$$

which are analytic in the open unit disk $U=\{z: z \in \mathcal{C}$ and $|z|<1\}$. We denote by $T$ the subclass of $A$ consisting of functions of the form

$$
f(z)=z-\sum_{n=2}^{\infty} a_{n} z^{n},\left(a_{n} \geq 0\right)
$$

This subclass was introduced and extensively studied by Silverman [17]. Let $T^{*}(\alpha)$ and $C(\alpha)$ be denote the subclasses of $T$ consisting of starlike and convex functions of order $\alpha,(0 \leq \alpha<1)$, respectively.

In [9], Kanas and Wisniowska introduced the classes $U C V(\alpha, \beta)$ consists of uniform $\beta$-convex functions of order $\alpha$ and $S P(\alpha, \beta)$ consists parabolic $\beta$-starlike functions of order $\alpha,-1<\alpha \leq 1, \beta \geq 0$, which generalizes the class $U C V$ and $S P$ respectively.

The function $f \in A$ belongs to $U C V(\alpha, \beta)$ if it satisfies the condition

$$
\operatorname{Re}\left\{1+\frac{z f^{\prime \prime}(z)}{f^{\prime}(z)}-\alpha\right\}>\beta\left\{\frac{z f^{\prime \prime}(z)}{f^{\prime}(z)}\right\}, \quad z \in U .
$$

The function $f \in A$ belongs to $S P(\alpha, \beta)$ if it satisfies the condition

$$
\operatorname{Re}\left\{\frac{z f^{\prime}(z)}{f(z)}-\alpha\right\}>\beta\left\{\frac{z f^{\prime}(z)}{f(z)}-1\right\}, \quad z \in U .
$$

Indeed, it follows from 1.3 and 1.4 that $f \in U C V(\alpha, \beta)$ if and only if $z f^{\prime}(z) \in S P(\alpha, \beta)$.

There has been a continues interest shown on the geometric and other related properties of Bessel functions (like hypergeometric function) after many papers have been published by Baricz [1] in recent times. One such problem of Baricz [2] was to find condition on the triple $p, b$ and $c$ such that the function $u_{p, b, c}$ is starlike and convex of order $\alpha$. In earlier investigations, finding conditions on the parameters for which the Gaussian hypergeometric functions belong to the various classes of functions have been discuss in details by Shanmugam [16], Sivasubramanina et al. [20].

Let us consider the following second order linear homogenous differential equation ( for more details see [3]) 
$(1.5) z^{2} \omega^{\prime \prime}(z)+b z \omega^{\prime}(z)+\left[c z^{2}-p^{2}+(1-b) p\right] \omega(z)=0, \quad(p, b, c \in \mathcal{C})$.

The function $\omega=\omega_{p, b, c}$ which is called the generalized Bessel function of the first kind of order $p$, is defined as a particular solution of 1.5. The function $\omega_{p, b, c}$ has the families representation as follows:

$$
\omega(z)=\omega_{p, b, c}(z)=\sum_{n=0}^{\infty} \frac{(-1)^{n} c^{n}}{n ! \Gamma\left(p+n+\frac{b+1}{2}\right)}\left(\frac{z}{2}\right)^{2 n+p}, z \in C,
$$

where $\Gamma$ stands for the Euler gamma function and $k=p+\frac{b+1}{2} \notin Z_{0}=$ $\{0,-1,-2, \cdots\}$. The series 1.6 permits the study of Bessel, modified Bessel, spherical Bessel, modified spherical Bessel and ultra spherical Bessel functions all together.

Solutions of 1.5 are referred as generalized Bessel functions of order $p$. The particular solution given by 1.6 is called the generalized Bessel function of the first kind of order $p$. Although the series defined above is convergent everywhere, the function $\omega_{p, b, c}$ is generally not univalent in the open unit $\operatorname{disc} U=\{z \in \mathcal{C}:|z|<1\}$.

It is worth mentioning that, in particular, for $b=c=1$ in 1.6, we obtain the familiar Bessel function of the first kind of order $p$ defined by ( see [3]),

$$
J_{p}(z)=\sum_{n=0}^{\infty} \frac{(-1)^{n}}{n ! \Gamma(p+n+1)}\left(\frac{z}{2}\right)^{2 n+p}, z \in C .
$$

For the choices of $b=1$ and $c=-1$ in 1.6, we obtain the modified Bessel function of the first kind order of $p$ defined by (see [3])

$$
I_{p}(z)=\sum_{n=0}^{\infty} \frac{1}{n ! \Gamma(p+n+1)}\left(\frac{z}{2}\right)^{2 n+p}, z \in C .
$$

Further, for the choices $b=2$ and $c=1$ in 1.6 , the functions $\omega_{p, b, c}$ reduces to $\sqrt{2} \frac{j_{p}}{\sqrt{\Pi}}$, where $j_{p}$ is the spherical Bessel function of the first kind of order $p$, defined by ( see [3])

$$
j_{p}(z)=\sqrt{\frac{\Pi}{2}} \sum_{n=0}^{\infty} \frac{(-1)^{n}}{n ! \Gamma\left(p+n+\frac{3}{2}\right)}\left(\frac{z}{2}\right)^{2 n+p}, z \in C .
$$

Deniz et al. [7] considered the function $u_{p, b, c}$ defined, in terms of generalized Bessel function $\omega_{p, b, c}$ by the transformation

$$
u_{p, b, c}(z)=2^{p} \Gamma\left(p+\frac{b+1}{2}\right) z^{1-\frac{p}{2}} \omega_{p, b, c}(\sqrt{z}) .
$$


By using the well known Pochhammer symbol (or the shifted factorial) $(\lambda)_{\mu}$ denoted, for $\lambda, \mu \in C$ and in terms of the Euler $\Gamma$-function, by

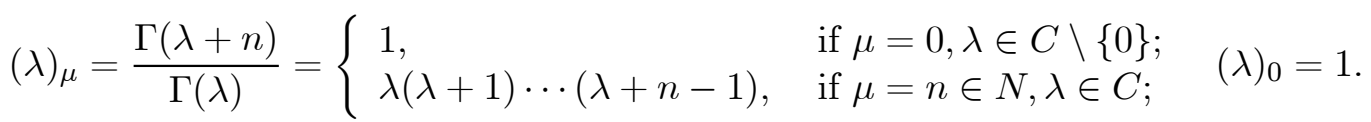

It is being understood conventionally that $(0)_{0}=1$, we obtain the following series representation for the function $u_{p, b, c}$ given by 1.10

$$
u_{p, b, c}(z)=z+\sum_{n=1}^{\infty} \frac{(-c)^{n}}{4^{n}(k)_{n} n !},
$$

where $k=p+\frac{b+1}{2} \notin Z$ and $N=\{1,2,3, \cdots\}$. For convenience, we write $u_{k, c}(z)=u_{p, b, c}(z)$.

For $f \in A$ is given by 1.1 and $g \in A$ is given by $g(z)=z+\sum_{n=1}^{\infty} b_{n} z^{n}$, the Hadamard product or convolution of $f(z)$ and $g(z)$ is defined by

$$
(f * g)(z)=z+\sum_{n=1}^{\infty} a_{n} b_{n} z^{n}=(g * f)(z), \quad z \in U .
$$

Note that $f * g \in A$. Now, we consider $B_{k}^{c}$ operator, which is defined as follows: $\mathrm{B}_{k}^{c} f(z)=u_{k, c}(z) * f(z)=z+\sum_{n=1}^{\infty} \frac{(-c)^{n} a_{n+1}}{4^{n}(k)_{n} n !} z^{n+1}$

$=z+\sum_{n=2}^{\infty} \frac{(-c)^{n-1} a_{n}}{4^{n-1}(k)_{n-1}(n-1) !} z^{n}=z+\sum_{n=2}^{\infty} D(c, k, n) a_{n} z^{n}$

where $D(c, k, n)=\frac{(-c)^{n-1}}{4^{n-1}(k)_{n-1}(n-1) !}, \quad k=\left(p+\frac{b+1}{2}\right) \neq 0,-1,-2, \cdots$. We note that using the definition 1.12, we obtain that

$$
z\left[B_{k+1}^{c} f(z)\right]^{\prime}=k B_{k}^{c} f(z)-(k-1) B_{k+1}^{c} f(z),
$$

where $k=p+\frac{b+1}{2} \notin Z_{0}^{-}$.

The function $B_{k}^{c} f(z)$ is in 1.12 is an elementary transformation of the generalized hypergeometric function, so that $B_{k}^{c} f(z)=z_{0} F_{1}\left(k ; \frac{-c}{4} z\right) * f(z)$ and $u_{k, c}\left(\frac{-c}{4} z\right)=z_{0} F_{1}(k ; z)$.

Recently, Baricz and Frasin [4] and Deniz et al. [7] were interested in the univalence of some integral operators which involved the normalization form of the ordinary Bessel function of the first kind and the normalized form of the generalized Bessel functions of the first kind, respectively.

Very recently, Cho et al. [6], Murugusundaramoorthy and Janani [13] and Porwal and Dixit [14] were introduced some characterization of generalized Bessel functions of first kind to be in certain subclasses of uniformly starlike and uniformly convex functions. 
Motivated by the new technique due to Ramachandran et al. [15], Murugusundaramoorthy and Magesh [11] and Thirupathi Reddy and Venkateswarlu [21], we define the following new subclass of uniformly convex functions defined by Bessel functions.

Definition 1.1. The function $f(z)$ of the form 1.1 is in the class $S(\lambda, \gamma)$ if it satisfies the inequality

$$
\operatorname{Re}\left\{\frac{z\left(B_{k}^{c} f(z)\right)^{\prime}}{(1-\lambda) z+\lambda B_{k}^{c} f(z)}-\gamma\right\}>\left|\frac{z\left(B_{k}^{c} f(z)\right)^{\prime}}{(1-\lambda) z+\lambda B_{k}^{c} f(z)}-1\right|
$$

for $0 \leq \lambda \leq 1,0 \leq \gamma<1$.

Further we denote $T S(\lambda, \gamma)=S(\lambda, \gamma) \cap T$.

The aim of this paper is to study some characterization for generalized Bessel functions of first kind is to be subclass of analytic functions. Further we studied coefficient estimates, radius of starlikeness, convexity, close-toconvexity. Convex linear combinations for the class $T S(\lambda, \gamma)$. Finally, we proved integral means inequalities for the class.

\section{Coefficient Estimates}

In this section we obtain the coefficient bounds of function $f(z)$.

Theorem 2.1. A function $f(z)$ of the form 1.1 is in $S(\lambda, \gamma)$, then

$$
\sum_{n=2}^{\infty}[2 n-\lambda(\gamma+1)] D(c, k, n)\left|a_{n}\right| \leq 1-\gamma
$$

where $0 \leq \lambda \leq 1,0 \leq \gamma<1$ and $D(c, k, n)$ is given by 1.12 .

Proof. It is enough to show that

$$
\left|\frac{z\left(B_{k}^{c} f(z)\right)^{\prime}}{(1-\lambda) z+\lambda B_{k}^{c} f(z)}-1\right|-R e\left\{\frac{z\left(B_{k}^{c} f(z)\right)^{\prime}}{(1-\lambda) z+\lambda B_{k}^{c} f(z)}-1\right\} \leq 1-\gamma .
$$

We have $\left|\frac{z\left(B_{k}^{c} f(z)\right)^{\prime}}{(1-\lambda) z+\lambda B_{k}^{c} f(z)}-1\right|-\operatorname{Re}\left\{\frac{z\left(B_{k}^{c} f(z)\right)^{\prime}}{(1-\lambda) z+\lambda B_{k}^{c} f(z)}-1\right\}$ $\leq 2\left|\frac{z\left(B_{k}^{c} f(z)\right)^{\prime}}{(1-\lambda) z+\lambda B_{k}^{c} f(z)}-1\right|$ 


$$
\begin{aligned}
& \leq \frac{2 \sum_{n=2}^{\infty}(n-\lambda) D(c, k, n)\left|a_{n}\right||z|^{n-1}}{1-\sum_{n=2}^{\infty} \lambda D(c, k, n)\left|a_{n}\right||z|^{n-1}} \\
& \leq \frac{2 \sum_{n=2}^{\infty}(n-\lambda) D(c, k, n)\left|a_{n}\right|}{1-\sum_{n=2}^{\infty} \lambda D(c, k, n)\left|a_{n}\right|} . \text { The last expression is bounded above by }(1-\gamma) \text { if } \\
& \sum_{n=2}^{\infty}[2 n-\lambda(\gamma+1)] D(c, k, n)\left|a_{n}\right| \leq(1-\gamma)
\end{aligned}
$$

and the proof is complete.

Theorem 2.2. Let $0 \leq \lambda \leq 1,0 \leq \gamma<1$. Then $f$ is in the form 1.2 to be in the class $T S(\lambda, \gamma)$ if and only if

$$
\sum_{n=2}^{\infty}[2 n-\lambda(\gamma+1)] D(c, k, n)\left|a_{n}\right| \leq(1-\gamma),
$$

where $D(c, k, n)$ is given by 1.12 .

Proof. In view of the above Theorem 2.1, we need only to prove the necessity. If $f \in T S(\lambda, \gamma)$ and $z$ is real then

$$
\operatorname{Re}\left\{\frac{1-\sum_{n=2}^{\infty} n D(c, k, n) a_{n} z^{n-1}}{1-\sum_{n=2}^{\infty} \lambda D(c, k, n) a_{n} z^{n-1}}\right\}>\left|\frac{\sum_{n=2}^{\infty}(n-\lambda) D(c, k, n) a_{n} z^{n-1}}{1-\sum_{n=2}^{\infty} \lambda D(c, k, n) a_{n} z^{n-1}}\right|
$$

Letting $z \rightarrow 1$ along the real axis, we desired the inequality

$$
\sum_{n=2}^{\infty}[2 n-\lambda(\gamma+1)] D(c, k, n)\left|a_{n}\right| \leq(1-\gamma)
$$

Corollary 2.1. If $f \in T S(\lambda, \gamma)$ then

$$
\left|a_{n}\right| \leq \frac{(1-\gamma)}{[2 n-\lambda(\gamma+1)] D(c, k, n)} z^{n}
$$

where $0 \leq \lambda \leq 1,0 \leq \gamma<1$ and $D(c, k, n)$ are given by 1.12. Equality holds for the function

$$
f(z)=z-\frac{(1-\gamma)}{[2 n-\lambda(\gamma+1)] D(c, k, n)} z^{n}
$$


Remark 2.2. For the choice of $\lambda=1$ in Theorems 2.1, 2.2 and Corollary 2.1, we observed that the results are coincide with Thirupathi Reddy and Venkateswarlu [21] .

\section{Convex Linear Combinations}

In this section we prove that the class $T S(\lambda, \gamma)$ is a convex set. And also we prove that if $f \in T S(\lambda, \gamma)$ then $f(z)$ is close-to-convex of order $\delta, 0 \leq \delta<1$.

Theorem 3.1. Let $f_{1}(z)=z$ and

$$
f_{n}(z)=z-\frac{(1-\gamma)}{[2 n-\lambda(\gamma+1)] D(c, k, n)} z^{n}, n \geq 2 .
$$

Then $f(z) \in T S(\lambda, \gamma)$ if and only if it can be in the form

$$
f(z)=\sum_{n=1}^{\infty} \omega_{n} f_{n}(z), \quad \omega_{n} \geq 0, \sum_{n=1}^{\infty} \omega_{n}=1
$$

Proof. Suppose that $f(z)$ can be written as in 2.6. Then

$$
f(z)=z-\sum_{n=2}^{\infty} \frac{(1-\gamma)}{[2 n-\lambda(\gamma+1)] D(c, k, n)} z^{n}
$$

Now $\sum_{n=2}^{\infty} \omega_{n} \frac{(1-\gamma)[2 n-\lambda(\gamma+1)] D(c, k, n)}{(1-\gamma)[2 n-\lambda(\gamma+1)] D(c, k, n)}=\sum_{n=2}^{\infty} \omega_{n}=\left(1-\omega_{1}\right) \leq 1$.

Thus $f(z) \in T S(\lambda, \gamma)$.

Conversely suppose that $f(z) \in T S(\lambda, \gamma)$. Then by using 2.3 , we get,

$$
\omega_{n}=\frac{[2 n-\lambda(\gamma+1)] D(c, k, n)}{1-\gamma} a_{n}, n \geq 2 \text { and } \omega_{1}=1-\sum_{n=2}^{\infty} \omega_{n}
$$

Then we have $f(z)=\sum_{n=1}^{\infty} \omega_{n} f_{n}(z)$. Hence the theorem.

Theorem 3.2. The class $T S(\lambda, \gamma)$ is a convex set. 
Proof. Let the function

$$
f_{j}(z)=z-\sum_{n=2}^{\infty} a_{n, j} z^{n}, \quad a_{n, j} \geq 0, j=1,2
$$

be in the class $T S(\lambda, \gamma)$. It is enough to show that the function $h(z)$ defined by $h(z)=\xi f_{1}(z)+(1-\xi) f_{2}(z), 0 \leq \xi<1$ is in the class $T S(\lambda, \gamma)$.

Since $h(z)=z-\sum_{n=2}^{\infty}\left[\xi a_{n, 1}+(1-\xi) a_{n, 2}\right] z^{n}$. An easy computation with the aid of Theorem 2.2, we get $\sum_{n=2}^{\infty}[2 n-\lambda(\gamma+1)] \xi D(c, k, n) a_{n, 1}+\sum_{n=2}^{\infty}[2 n-$ $\lambda(\gamma+1)](1-\xi) D(c, k, n) a_{n, 2}$ $\leq \xi(1-\gamma)+(1-\xi)(1-\gamma) \leq(1-\gamma)$, which implies that $h \in T S(\lambda, \gamma)$. Hence the $T S(\lambda, \gamma)$ is convex. Now we obtain the radii of close-to-convexity starlikeness and convexity for the class $T S(\lambda, \gamma)$.

Theorem 3.3. Let the functin $f(z)$ defined by 1.2 belong to the class $T S(\lambda, \gamma)$. Then $f(z)$ is close-to-convex of order $\delta,(0 \leq \delta<1)$ in the disc $|z|<r_{1}$, where

$$
r_{1}=\inf _{n \geq 2}\left[\frac{(1-\delta) \sum_{n=2}^{\infty}[2 n-\lambda(\gamma+1)] D(c, k, n)}{n(1-\gamma)}\right]^{\frac{1}{n-1}}, \quad n \geq 2 .
$$

The result is sharp with the extremal function $f(z)$ by 2.5 .

Proof. Given $f \in T$ and $f$ is close-to-convex of order $\delta$, we have

$$
\left|f^{\prime}(z)-1\right|<(1-\delta)
$$

For the left hand side of 2.9 , we have $\left|f^{\prime}(z)-1\right| \leq \sum_{n=2}^{\infty} n a_{n}|z|^{n-1}$.

The right hand side of the above inequality is less than $(1-\delta)$. Then

$$
\sum_{n=2}^{\infty} \frac{n}{1-\delta} a_{n}|z|^{n-1} \leq 1
$$

Using the fact that, $f(z) \in T S(\lambda, \gamma)$ if and only if $\sum_{n=2}^{\infty} \frac{[2 n-\lambda(\gamma+1)] D(c, k, n)}{(1-\gamma)} a_{n} \leq$ 1.

We can 2.9 is true if

$$
\frac{n}{1-\delta}|z|^{n-1} \leq \frac{[2 n-\lambda(\gamma+1)] D(c, k, n)}{(1-\gamma)}
$$


or equivalently $|z| \leq\left[\frac{(1-\delta)[2 n-\lambda(\gamma+1)] D(c, k, n)}{n(1-\gamma)}\right]^{\frac{1}{n-1}}$ and hence the proof of the theorem.

Theorem 3.4. Let the function $f(z)$ defined by 1.2 belong to the class $T S(\lambda, \gamma)$. Then $f(z)$ is starlike of order $\delta,(0 \leq \delta<1)$ in the disc $|z|<r_{2}$, where

$$
r_{2}=\inf _{n \geq 2}\left[\frac{(1-\delta) \sum_{n=2}^{\infty}[2 n-\lambda(\gamma+1)] D(c, k, n)}{(n-\delta)(1-\gamma)}\right]^{\frac{1}{n-1}}, \quad n \geq 2 .
$$

The result is sharp with the extremal function given by 2.4

Proof. Given $f \in T$ and $f$ is starlike of order $\delta$, we have

$$
\left|\frac{z f^{\prime}(z)}{f(z)}-1\right|<(1-\delta)
$$

For the left hand side of 2.11, we have $\left|\frac{z f^{\prime}(z)}{f(z)}-1\right| \leq \frac{\sum_{n=2}^{\infty}(n-1) a_{n}|z|^{n-1}}{1-\sum_{n=2}^{\infty} a_{n}|z|^{n-1}}$.

The right hand side of the above inequality is less than $(1-\delta)$ if

$$
\sum_{n=2}^{\infty} \frac{(n-\delta)}{(1-\delta)} a_{n}|z|^{n-1}<1
$$

Using the fact that $f(z) \in T S(\lambda, \gamma)$ if and only if $\sum_{n=2}^{\infty} \frac{[2 n-\lambda(\gamma+1)] D(c, k, n)}{(1-\gamma)} a_{n} \leq$ 1 ,

2.11 is true if $\frac{(n-\delta)}{(1-\delta)}|z|^{n-1} \leq \frac{[2 n-\lambda(\gamma+1)] D(c, k, n)}{(1-\gamma)}$

or equivalently $|z|^{n-1} \leq \frac{(1-\delta)[2 n-\lambda(\gamma+1)] D(c, k, n)}{(n-\delta)(1-\gamma)}$.

It yield starlikeness of the family.

Remark 3.1. For the choice of $\lambda=1$ in Theorems 3.1, 3.2, 3.3 and 3.4, we observed that the results are coincide with Thirupathi Reddy and Venkateswarlu [21] . 


\section{Integral Means Inequalities}

In [18], Silverman found that the function $f_{2}(z)=z-\frac{z^{2}}{2}$ is often extremal over the family $T$ and applied this function to resolve his integral means inequality, conjectured [18] and settled in [19], that

$$
\int_{0}^{2 \Pi}\left|f\left(r e^{i \phi}\right)\right|^{\eta} d \theta \leq \int_{0}^{2 \Pi}\left|f_{2}\left(r e^{i \phi}\right)^{\eta}\right| d \theta,
$$

for all $f \in T, \eta>0$ and $0<r<1$. In [18], he also proved his conjecture for the subclasses $T^{*}(\alpha)$ and $C(\alpha)$ of $T$.

Now we prove Silverman's conjecture for the class of functions $T S(\lambda, \gamma)$. We need the concept of subordination between analytic functions and a subordination theorem of Littlewood [10]. Two functions $f$ and $g$, which are analytic in $E$, the function $\omega$ is said to be subordinate to $g$ in $E$ if there exists a function $\omega$ analytic in $E$ with $\omega(0)=0,|\omega(z)|<1,(z \in E)$ such that $f(z)=g(\omega(z)),(z \in E)$. We denote this subordination by $f(z) \prec g(z)$.

Lemma 4.1. [10] If the functions $f$ and $g$ are analytic in $E$ with $f(z) \prec$ $g(z)$, then for $\eta>0$ and $z=r e^{i \phi}, 0<r<1, \int_{0}^{2 \Pi}\left|g\left(r e^{i \phi}\right)\right|^{\eta} d \theta \leq \int_{0}^{2 \Pi}\left|f\left(r e^{i \phi}\right)\right|^{\eta} d \theta$.

Now, we discuss the integral means inequalities for functions $f \in T S(\lambda, \gamma)$ and $\int_{0}^{2 \Pi}\left|g\left(r e^{i \phi}\right)\right|^{\eta} d \theta \leq \int_{0}^{2 \Pi}\left|f\left(r e^{i \phi}\right)\right|^{\eta} d \theta$.

Theorem 4.1. Suppose $f(z) \in T S(\lambda, \gamma), \eta>\theta, 0 \leq \lambda<1,0 \leq \gamma<1$ and $f_{2}(z)$ be defined by

$$
f_{2}(z)=z-\frac{1-\gamma}{\phi_{2}(\lambda, \gamma)} z^{2}
$$

where $\phi_{2}=[4-\lambda(\gamma+1)] D(c, k, 2)$ and $D(c, k, 2)$ is given by 1.12. Then $z=r e^{i \theta}, 0<r<1$,

$$
\int_{0}^{2 \Pi}|f(z)|^{\eta} d \theta \leq \int_{0}^{2 \Pi}\left|f_{2}(z)\right|^{\eta} d \theta .
$$


Proof. For $f(z)=z-\sum_{n=2}^{\infty} a_{n} z^{n}, 2.13$ is equivalent to proving that

$$
\int_{0}^{2 \Pi}\left|1-\sum_{n=2}^{\infty}\right| a_{n}\left|z^{n-1}\right|^{\eta} d \theta \leq \int_{0}^{2 \Pi}\left|1-\frac{1-\gamma}{\phi_{2}(\lambda, \gamma)} z\right|^{\eta} d \theta .
$$

By Lemma 4.1, it is enough to prove that $1-\sum_{n=2}^{\infty} a_{n} z^{n-1} \prec 1-\frac{1-\gamma}{\phi_{2}(\lambda, \gamma)} z$.

$$
\text { Setting } 1-\sum_{n=2}^{\infty} a_{n} z^{n-1} \prec 1-\frac{1-\gamma}{\phi_{2}(\lambda, \gamma)} \omega(z)
$$

and using 2.2, we obtain $\omega(z)$ is analytic in $U, \omega(z)=0$ and if

$$
|\omega(z)|=\left|\sum_{n=2}^{\infty} \frac{\phi_{2}(\lambda, \gamma)}{1-\gamma}\right| a_{n}\left|z^{n-1}\right| \leq|z| \sum_{n=2}^{\infty} \frac{\phi_{n}(\lambda, \gamma)}{1-\gamma}\left|a_{n}\right| \leq|z|,
$$

where $\phi_{n}(\lambda, \gamma)=[2 n-\lambda(\gamma+1)] D(c, k, n)$.

Hence the proof is completed.

Remark 4.2. For the choice of $\lambda=1$ in Theorem 4.1, we observed that the result is coincide with Thirupathi Reddy and Venkateswarlu [21] .

\section{Acknowledgements}

The authors would like to express sincere thanks to the esteemed Referee(s) for their careful readings, valuable suggestions and comments, which helped them to improve the presentation of this paper.

\section{References}

[1] A. Baricz, "Geometric properties of generalized Bessel function", Publicationes Mathematicae, vol. 73, no. 1-2, pp. 155-178, 2008. [On line]. Available: https://bit.ly/20vSxcO

[2] A. Baricz, Generalized Bessel functions of the first kind, Ph. D. thesis, Babes-Bolyai University, Cluj-Napoca, Rumania, 2008.

[3] A. Baricz, Generalized Bessel functions of the first kind, vol. 1994. Heidelberg: Springer, 2010, doi: 10.1007/978-3-642-12230-9 
[4] A. Baricz and B. Frasin, "Univalence of integral operators involving Bessel functions", Applied mathematics letters, vol. 23, no. 4, pp. 371376, Apr. 2010, doi: 10.1016/j.aml.2009.10.013

[5] A. Baricz, E. Deniz, M. Caglar and H. Orhan, "Differential subordinations involving generalized Bessels functions", Bulletin of the malaysian mathematical sciences society, vol. 38, no. 3, pp. 1255-1280, Jul. 2015, doi: 10.1007/s40840-014-0079-8.

[6] N. Cho, H. Lee and R. Srivastava, "Characterizations for certain subclasses of starlike and convex functions associated with Bessel functions", Filomat, vol. 30, no. 7, pp. 1911-1917, 2016. [On line]. Available: https://bit.ly/312bfeX

[7] E. Deniz, H. Orhan and H. Srivastava, "Some sufficient conditions for univalence of certain families of integral operators involving generalized Bessel functions", Taiwanese journal of mathematics, vol. 15, no. 2, pp. 883-917, Apr. 2011, doi: 10.11650/twjm/1500406240

[8] E. Deniz, "Convexity of integral operators involving generalized Bessel functions", Integral transforms and special functions, vol. 24, no. 3, pp. 201-216, May 2013, doi: 10.1080/10652469.2012.685938.

[9] S. Kanas and A. Wisniowska, "Conic regions and K-uniform convexity", Journal of computational and applied mathematics, vol. 105, no. 1-2, pp. 327-336, May 1999, doi: 10.1016/S0377-0427(99)00018-7.

[10] J. Littlewood, "On inequalities in the theory of functions", Proceeding of the London mathematical society, Vol. s2-23, no. 1, pp. 481-519, 1925, doi: 10.1112/plms/s2-23.1.481.

[11] G. Murugusundaramoorthy and N. Magesh, "On certain subclasses of analytic functions associated with hypergeometric functions", Applied mathematics letters, vol. 24, no. 4, pp. 494-500, Apr. 2011, doi: 10.1016/j.aml.2010.10.048.

[12] G. Murugusundaramoorthy, K. Vijaya and M. Kasturi, A note on subclasses of starlike and convex functions associated with Bessel functions, J. Nonlinear Funct. Anal., vol. 2014, 11, Apr. 2014. [On line]. Available: https://bit.ly/2LYanDE

[13] G. Murugusundaramoorthy and T. Janani, "An application of generalized Bessel functions on certain subclasses of analytic functions", Turkish journal of analysis and number theory, vol. 3, no. 1, pp. 1-6, 2015, doi: 10.12691/tjant-3-1-1.

[14] S. Porwal and K. Dixit, "An application of generalized Bessel functions on certain analytic functions", Acta Universitatis matthiae belii series mathematics, vol. 21, pp. 51-57, 2013. [On line]. Available: https://bit.ly/311j6tg

[15] C. Ramachandran, K. Dhanalakshmi and V. Lakshminarayanan, "Certain aspects of univalent function with negative coefficients defined by Bessel function", Brazilian archives of biology and technology, vol. 59, no. special 2, pp. 1-14, 2016, doi: 10.1590/1678-43242016161044. 
[16] T. Shanmugam, "Hypergeometric functions in the geometric function theory", Applied mathematics computation, vol. 187, no. 1, pp. 433444, Apr. 2007, doi: 10.1016/j.amc.2006.08.143.

[17] H. Silverman, "Univalent functions with negative coefficients", Proceedings of the American mathematical society, vol. 51, pp. 109-116, 1975, doi: 10.1090/S0002-9939-1975-0369678-0.

[18] H. Silverman, "A survey with open problems on univalent functions whose coefficient are negative", Rocky Mountain journal of mathematics, vol. 21, no. 3, pp. 1099-1125, 1991, doi: $10.1216 / \mathrm{rmjm} / 1181072932$.

[19] H. Silverman, "Integral means for univalent functions with negative coefficient", Houston journal of mathematics, vol. 23, no. 1, pp. 169174, 1997. [On line]. Available: https://bit.ly/3270qI0

[20] S. Sivasubramanian, T. Rosy and K. Muthunagai, "Certain sufficient conditions for a subclass of analytic functions involving Hohlov operator", Computers \& mathematics with applications, vol. 62, no. 12, pp. 4479-4485, Dec. 2011, doi: 10.1016/j.camwa.2011.10.025.

[21] P. Thirupathi and B. Venkateshwarlou, "On a certain subclass of uniformly convex functions defined by Bessel functions", Transylvanian journal of mathematics and mechanics, vol. 10, no. 1, pp. 43-49, 2018. [On line]. Available: https://bit.ly/3118AlB 